Review Article

\title{
Regional Cerebral Blood Flow in Mild Cognitive Impairment and Alzheimer's Disease Measured with Arterial Spin Labeling Magnetic Resonance Imaging
}

\author{
Alba Sierra-Marcos ${ }^{1,2}$ \\ ${ }^{1}$ Department of Neurology, Barcelona Medicine and Surgery Institute (IMECBA), Barcelona, Spain \\ ${ }^{2}$ Alzheimer Disease Research Unit, CIEN Foundation, Queen Sofia Foundation Alzheimer Center, Madrid, Spain
}

Correspondence should be addressed to Alba Sierra-Marcos; asierra@neurologiabarcelona.es

Received 16 January 2017; Revised 16 February 2017; Accepted 22 February 2017; Published 1 March 2017

Academic Editor: Francesco Panza

Copyright (C) 2017 Alba Sierra-Marcos. This is an open access article distributed under the Creative Commons Attribution License, which permits unrestricted use, distribution, and reproduction in any medium, provided the original work is properly cited.

\begin{abstract}
Alzheimer's disease (AD) depicts dynamic changes in regional brain function from early stages of the disease. Arterial spin labeling(ASL-) based MRI methods have been applied for detecting regional cerebral blood flow (rCBF) perfusion changes in patients with AD and mild cognitive impairment (MCI). Nevertheless, the results obtained from ASL studies in AD and MCI are still controversial, since rCBF maps may show both hypoperfusion or hyperperfusion areas in brain structures involved in different cognitive functions. The goal of this review is to provide the current state of the art regarding the role of ASL for detecting distinctive perfusion patterns in subjects with $\mathrm{MCI}$ and/or AD. The ability to obtain this information using a noninvasive and widely available modality such as ASL should greatly enhance the knowledge into the broad range of hemodynamically related changes taking place during the cognitive decline process in $\mathrm{AD}$.
\end{abstract}

\section{Introduction}

Alzheimer's disease $(\mathrm{AD})$ is a devastating neurodegenerative disorder with an important socioeconomic impact [1]. Mild cognitive impairment (MCI) is considered the prodromal phase of $\mathrm{AD}$, and it is characterized by early cognitive symptoms not severe enough to meet the criteria for dementia [2]. With the prospect of disease-modifying therapies, it is desirable to detect signs of neurodegeneration at an earlier stage of the disease, before neuronal cell destruction is detectable at structural MR imaging as atrophy. Changes in regional brain function may be more dynamic and provide even greater sensitivity to early disease, disease progression, or responses to therapy than changes in regional gray matter volume depicted by structural MRI $[3,4]$.

Arterial spin labeling- (ASL-) based MRI methods have been applied for detecting regional cerebral blood flow (CBF) perfusion changes in patients with AD or MCI [5-16]. Since the inception of this technique more than 20 years ago [17], the quality of ASL-derived perfusion maps has reached a level that makes the method useful for many clinical and research applications. Important advantages of this technique are its noninvasiveness (since magnetically labeled protons in blood are used as a tracer) and short acquisition time at high magnetic field strengths $(3.0 \mathrm{~T})$ [18]. ASL has been validated extensively against other methods that use exogenous contrast agents, such as Positron Emission Tomography (PET) [19], and ASL implementation are now commercially available on all major MRI platforms, with demonstrated reproducibility in multicenter studies [20,21]. The ability to quickly and noninvasively obtain CBF maps should greatly enhance the understanding of the hemodynamic mechanisms related to neurodegeneration [17, 22]. Since MRI scanners are more widely available than PET scanners (and also less expensive), ASL might become an alternative for FDG-PET [23, 24], allowing a higher throughput of patients. However, an important drawback for the implementation of ASL in clinical settings has been the lack of standardized protocols. Recently, international guidelines [25] and consensual initiatives have been developed to account for this issue. 
Nevertheless, the results obtained from ASL studies in AD and MCI are still controversial. The goal of this review is to provide the current state of the art regarding the role of ASL for detecting distinctive perfusion patterns in subjects with $\mathrm{MCI}$ and/or AD.

\section{A Brief Overview of the ASL Technique}

ASL technique was developed in the 90s, using electromagnetically labeled arterial water as a standard diffusible tracer method for measuring perfusion. Proximal ASL alters the total magnetization of the protons in the arterial water of the distal tissue, and the extent of this alteration is determined by comparison of labeling images with control images. With an additional knowledge of the regional T1 of tissue, quantitative blood flow maps can be generated, since the electromagnetically labeled tracer has a decay rate of T1, allowing perfusion of the tissue and microvasculature to be detected [26].

The existing ASL techniques can be sorted out in two categories: "continuous (CASL)" or "pseudocontinuous (PCASL)" and "pulsed (PASL)" techniques, depending on the spatial extent and the duration of the spin labeling. In CASL/PCASL, labeling occurs as blood flows through a single labeling plane over a long period (1-3 s), whereas in PASL a slab of tissue, including arterial blood flow, is labeled using a single short radiofrequency pulse or limited number of them, with a total duration of $10-20 \mathrm{~ms}$. The labeling efficiency and the ease of the incorporation into a clinical setting are superior with PASL, whereas the SNR is superior with CASL.

\section{An Overview of the Literature in ASL and MCI/Dementia}

Current knowledge regarding ASL hypo/hyperperfusion patterns in AD is still controversial. An important drawback of available studies is the heterogeneity of participant's demographics, clinical diagnosis (involving cognitively healthy elderly population, different types of MCI, AD, other dementia types, etc.), neuropsychological tools implemented for evaluation, and the methodology used for measuring CBF. In summary, it is not possible to precisely compare studies with different population subsets and nonreplicable methods.

Globally, regional CBF maps depicted by ASL may show both hypoperfusion or hyperperfusion areas at a global or localized scale, located in brain structures involved in different cognitive functions (Table 1).

More in detail, patients suffering from MCI depict areas of hypoperfusion in bilateral parietal lobes [7], posterior cingulate cortex (PCC) and precuneus [10, 13], left (L) occipital lobe, and bilateral frontal and temporal lobes [14], compared to healthy controls (HC). Interestingly, one study also found an increase in perfusion in the L hippocampus, right (R) amygdala, and basal ganglia (including rostral head of the $\mathrm{R}$ caudate nucleus, ventral putamen, and globus pallidus) [10].

Considering patients with fully developed $\mathrm{AD}$, hypoperfusion areas are also present in the PCC and precuneus, bilateral parietal and temporal lobes, and bilateral superior and middle frontal gyri $[6-8,10,12-14]$. Additionally, temporooccipital and parietooccipital association cortices [5], L limbic lobe [14], and L orbitofrontal cortex [10] also depicted a hypoperfusion in certain studies $[5-7,9,10,12-$ $15,28]$. Similarly to MCI, an increase in perfusion has been shown in the hippocampus $[9,11]$ and basal ganglia (putamen, caudate, lentiform, and thalamus) [14]. Additionally, anterior cingulate cortex (ACC) [10] and R limbic lobe [14] have also demonstrated a hyperperfusion in $\mathrm{AD}$ patients. Conversely, Asllani et al. [8] and Bron et al. [15] reported a hypoperfusion in medial temporal lobe structures $[8,15]$. All abovementioned studies correspond to cross-sectional analysis comparing a pathological condition with a cognitively healthy state. Additionally, there is one longitudinal study including two groups of healthy patients at baseline, one of those exhibiting cognitive decline in follow-up visits. The "converter" group depicted a hypoperfusion in the PCC already at baseline [16], demonstrating that ASL might be a useful tool for predicting conversion to disease in the healthy elderly.

Additionally, nonsymptomatic high-risk groups may depict a hyperperfusion in the hippocampus, as individuals with a parental history of AD and at least one copy of APOE4 [11].

Finally, most of these studies have explored ASL in a resting state. Additionally, there are two studies using a memoryencoding task during the acquisition. When comparing task to baseline, patients with amnestic MCI [29] or at risk of $\mathrm{AD}$ (due to their APOE4 genotype and family history) [11] showed reduced activation in the parahippocampal gyrus [29] and hippocampus [11], compared to controls. It is relevant that these patients at risk of $\mathrm{AD}$ presented an elevated perfusion in the hippocampus at baseline [11], which might suggest that individuals with $\mathrm{MCI}$ or $\mathrm{AD}$ may lack the dynamic capability to modulate regional CBF in response to task demands.

From these studies we can extract that MCI and AD depict similar regions of perfusion changes, in particular more or less restricted areas of hypoperfusion in the PCC, precuneus, and other parietal regions, along with areas of hyperperfusion in the middle temporal lobe in some studies. In particular, the identification of a hypoperfusion in the PCC and precuneus had a sensitivity/specificity of 91\%/80\% [28], whereas using a combination of different regions in subjects with amnestic and dysexecutive MCI the accuracy was 60$70 \%[30]$.

Otherwise, the combination of structural and functional imaging in the same setting might contribute to the diagnosis of $\mathrm{AD}$ in clinical practice, as demonstrated in a study in which T1 weighted structural scans had 56/70\% sensitivity/specificity in the detection of mild $\mathrm{AD}$, while adding qualitative analysis of ASL increased these percentages to $85 / 54 \%$ [4].

Overall, the most consistent finding thorough the literature is a hypoperfusion in the PCC, which has been detected in all stages of AD (pre-MCI, MCI, and fully developed AD). Taking into account this piece of information, this finding might be considered as a functional biomarker of the disease. 


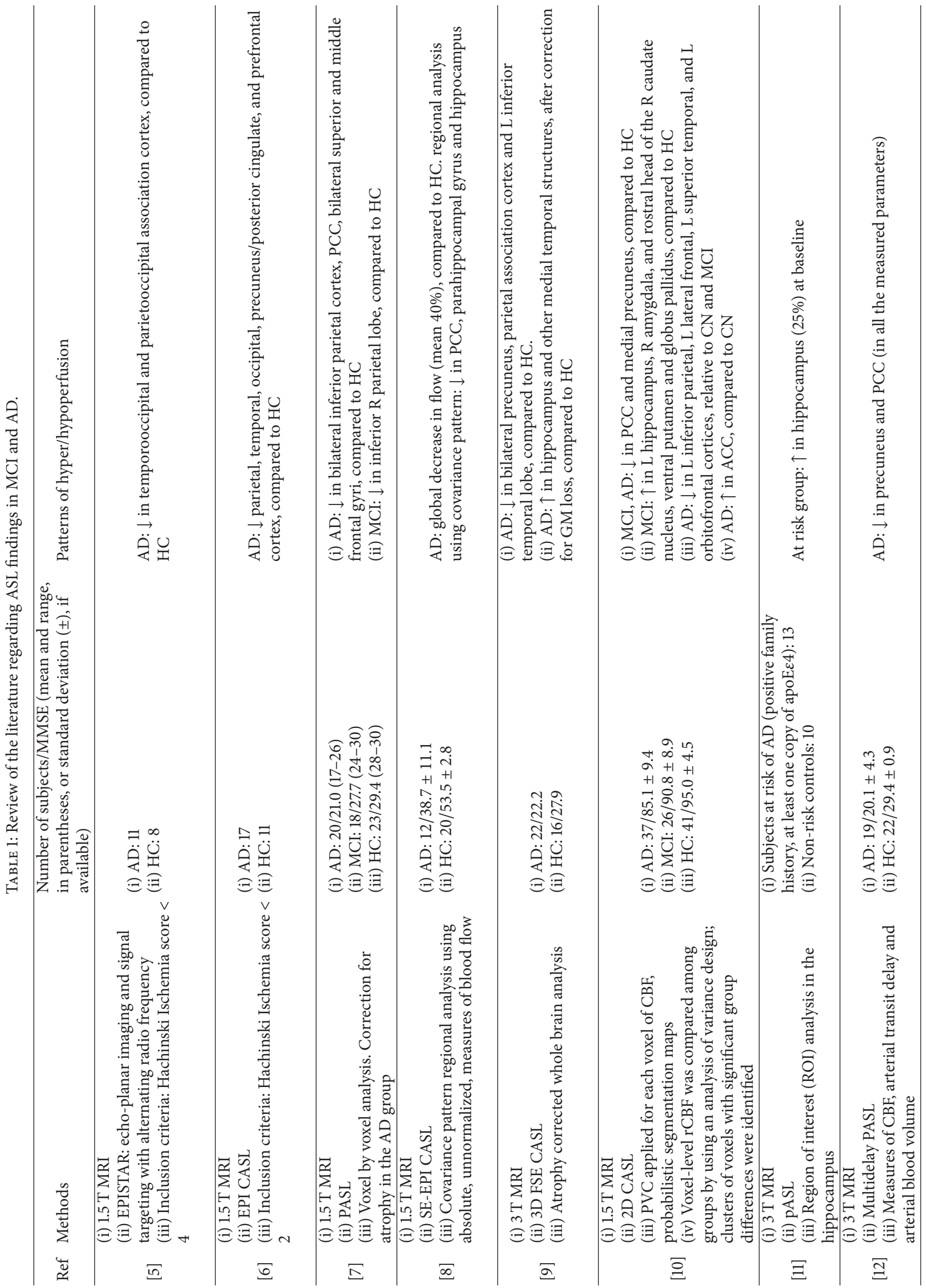




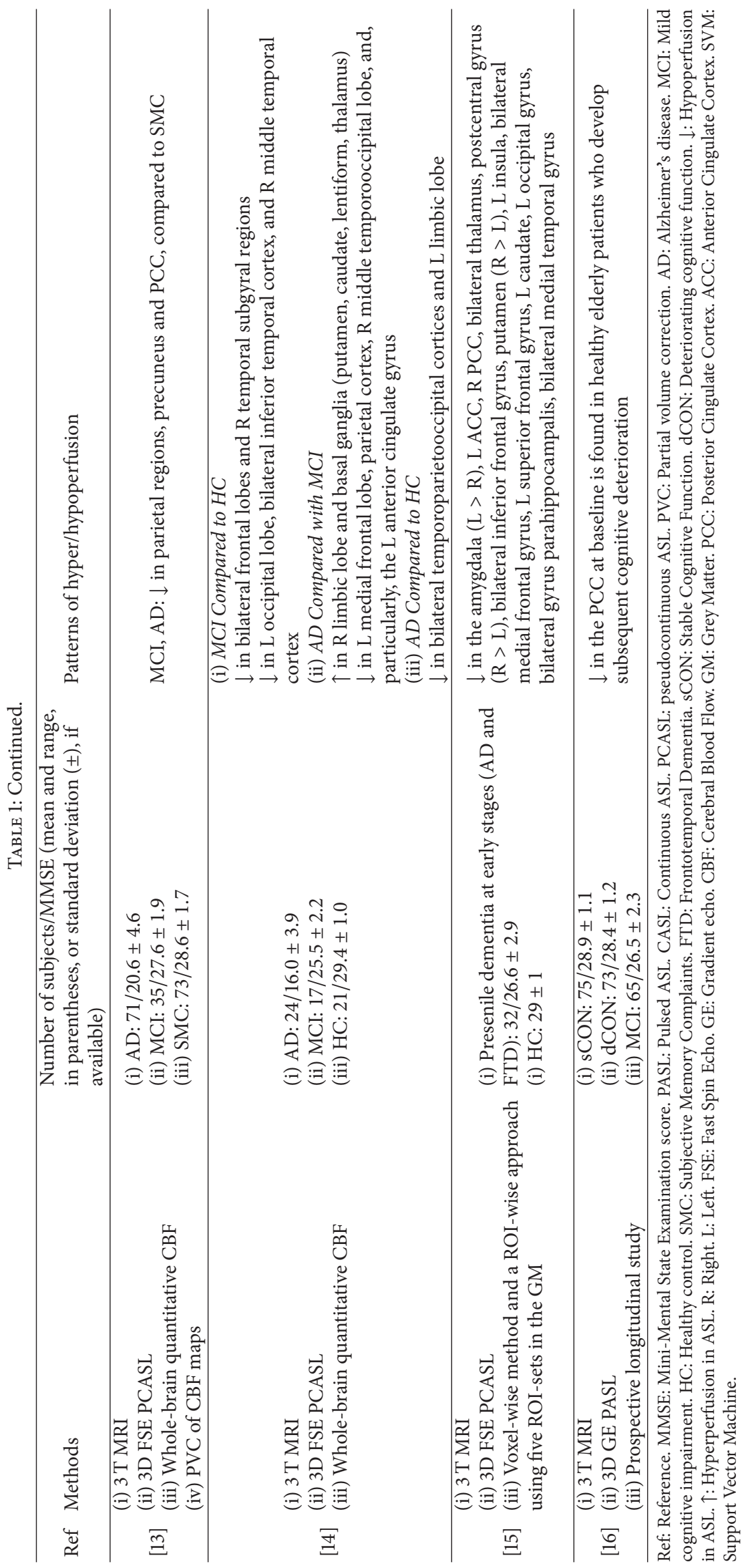




\section{Physiological Interpretation}

Considering these studies, different patterns of CBF might coexist or evolve with the progression of $\mathrm{AD}$. In early stages of the disease, certain areas such as the prefrontal cortex [31] or medial temporal lobe structures would eventually have the capability for "compensating" the cognitive decline, depicting an elevated atrophy-corrected perfusion. This compensatory mechanism could be explained by a pathological elevation of neural activity, release of inflammatory molecules, and/or increased blood supply through vascular dilation or increased vascular density $[9,32]$. Simultaneously, perfusion deficits might be also present from the very early preclinical phases of $\mathrm{AD}$, persisting into the latest stages of the disease, demonstrating a progressive hypoperfusion with disease development, leading to serious consequences on brain structure, cognition, and function in advanced stages of the disease.

Regarding the physiological interpretation of the regional hypoperfusion consistently found in the PCC, precuneus and/or lateral parietal cortex, a debate exists as to whether this is a cause or consequence of the disease [33]. It is known that vascular factors such as ischemic stroke, atherosclerosis, hypertension, diabetes, and cardiac disease are repeatedly implicated in the risk of developing $\mathrm{AD}$ [34]. While $\mathrm{AD}$ and vascular dementia have traditionally been considered as distinct entities, there is growing evidence of an overlap between these categories [35]. Furthermore, perfusion measures in the parietooccipital region [5] and parietal cortex along with the precuneus/posterior cingulate [6] have been shown to correlate with dementia severity (measured with the Blessed Dementia Scale [5] and MMSE [6]). Hypoperfusion may lead to changes in cortical thickness, as obtained from structural MRI scans, in areas most vulnerable to aging (medial prefrontal and pericentral cortices) as well as in areas associated with amyloid-aggregation (e.g., occipitotemporal and basal temporal cortices), especially in APOE4 carriers [36]. Finally, evidence from aging and stroke studies suggests that chronic brain hypoperfusion leads to neurodegeneration and cognitive impairment [33].

A hyperperfusion in medial temporal lobe structures has also been found with less consistency. The hypothesis of an increase of neural activity in these regions has been supported by two clinical studies [37, 38]. Choline acetyltransferase (ChAT), the enzyme responsible for synthesis of acetylcholine, was decreased in the hippocampi of patients suffering from MCI, whereas this elevation was no longer present in mild $\mathrm{AD}$ cases, and severe $\mathrm{AD}$ patients had markedly depleted levels. Cholinergic system might be capable of compensatory responses during early stages of dementia, so memory impairment in MCI might be the result of a progressive loss of entorhinal input to hippocampus, rather than a loss in hippocampal neural activity.

Additionally, alterations in the blood brain barrier and brain vasculature are known to be involved in neurodegeneration and neuroinflammation processes in $\mathrm{AD}$. It has been suggested that a dynamic cycle of angiogenic events contributes to $A \beta$ accumulation and neuronal death [39], and simultaneously amyloid- $\beta(\mathrm{A} \beta)$ plaques and neurofibrillary tangles (NFT), the pathological lesions found in the mesial temporal and neocortex of $\mathrm{AD}$ brains [40], may also be angiogenic [41, 42]. In a postmortem study comparing $\mathrm{AD}$ with $\mathrm{HC}$ brain tissues, the hippocampus was the only brain region exhibiting both angiogenic vessels (demonstrated using integrin $\alpha \mathrm{v} \beta 3$ immunoreactivity) and increased vascular density [32]. This is consistent with the studies performed in APP23 transgenic animal models of $\mathrm{AD}$, where $\beta 3$ positive vessels were increased in disease susceptible regions [43], and also with studies linking $\mathrm{AD}$ to inflammatory mediators (TNF $\alpha$, IL1 $\beta$, IL-6, and IL-8), all of them with proangiogenic properties $[44,45]$, and with in vitro studies showing that microvessels of $\mathrm{AD}$ brains release angiogenic mediators such as angiopoietin-2 and vascular endothelial growth factor (VEGF) [46]. Finally, animal models have also shown that cognitive decline is enhanced following endothelial cell activation, proliferation, and subsequent vascular remodeling [43].

In conclusion, $\mathrm{AD}$ seems to behave as a dynamic process mediated by aberrant angiogenesis and inflammation mechanisms acting in certain vulnerable areas, leading to further neurodegeneration and progressive cognitive decline.

\section{What Can ASL Add to the Standard Brain Perfusion Imaging Techniques?}

In $\mathrm{AD}$, perfusion ( $\mathrm{CBF}$ and cerebral blood volume (CBV)) has been measured using a number of different imaging modalities, including MRI, xenon enhanced X-ray computed tomography (XeCT), or Single Photon Emission Computed Tomography (SPECT). Otherwise, PET studies provided the first maps of activation patterns in the human brain with high spatial resolution, by measuring changes energy metabolism [47]. Since then, numerous imaging techniques have been developed and applied to evaluate brain function [27], using different tracers (diffusible or nondiffusible, endogenous or exogenous), with different technical requirements. Brain perfusion imaging techniques also differ in terms of the duration of data acquisition, processing, quantitative accuracy, brain coverage, and spatial resolution, giving rise to the strengths and weaknesses of each technique for detecting alterations in neurodegenerative disorders (Table 2). For the past two decades, the nuclear medicine techniques SPECT and FDGPET have served as the mainstream imaging techniques for perfusion and metabolism studies in AD. SPECT, however, has a low spatial resolution (around $1 \mathrm{~cm}$ ), whereas FDGPET has a $5 \mathrm{~mm}$ resolution and a higher sensitivity in low perfusion areas [31]. These techniques, however, require the use of exogenous radioactive tracers and are more expensive than the more recently developed perfusion-weighted MRI (PW-MRI) technique. Currently, PW-MRI is the most widely available technique for evaluating brain perfusion, by means of the dynamic susceptibility contrast (DSC) approach, which detects the first passage of an intravascular contrast agent such as gadolinium chelate, and the ASL technique, which uses magnetically labeled arterial blood water as a diffusible endogenous tracer. These two approaches measure different aspects of the perfusion state of the tissue. The contrast agent techniques provide a robust measurement of $\mathrm{CBV}$, whereas 
TABLE 2: Overview of the imaging techniques dedicated to brain hemodynamics (adapted from Wintermark et al. [27]).

\begin{tabular}{|c|c|c|c|c|c|}
\hline & PET & SPECT & DSC & ASL & fMRI \\
\hline Age range & $\begin{array}{l}\text { Adults (and children } \\
\text { for static exams) }\end{array}$ & Adults (and children) & Adults (and children) & Adults + children & Adults (and children) \\
\hline Contrast material & ${ }^{15} \mathrm{O}_{2}, \mathrm{C}^{15} \mathrm{O}_{2}, \mathrm{H}_{2}{ }^{15} \mathrm{O}$ & $\begin{array}{c}{ }^{99} \mathrm{Tc}-\mathrm{HMPAO}, \\
{ }^{99} \mathrm{Tc}-\mathrm{ECD},{ }^{133} \mathrm{Xe}, \\
{ }^{123} \mathrm{I} \text {-IMP (diffusible) }\end{array}$ & $\begin{array}{l}\text { Gadolinium chelate } \\
\text { (nondiffusible) }\end{array}$ & $\begin{array}{l}\text { None (endogenous } \\
\text { contrast) }\end{array}$ & None \\
\hline Radiation/study & $0.5-2 \mathrm{mSv}$ & $3.5-12 \mathrm{mSv}$ & None & None & None \\
\hline Data acquisition & $5-9 \min$ & $10-15 \min$ & $1 \mathrm{~min}$ & $5-10 \mathrm{~min}$ & \\
\hline Data processing & $5-10 \mathrm{~min}$ & $5 \mathrm{~min}$ & $5 \mathrm{~min}$ & $5 \mathrm{~min}$ & \\
\hline Assessed parameters & $\begin{array}{l}\text { CBV, CBF, rOEF, } \\
\text { glucose metabolism }\end{array}$ & $\mathrm{CBF}$ & $\begin{array}{l}\text { CBF, CBV, MTT, TTP, } \\
\text { permeability map }\end{array}$ & $\mathrm{CBF}$ & BOLD signal \\
\hline Quantitative accuracy & Yes & $\begin{array}{l}\text { Yes for }{ }^{133} \mathrm{Xe} \text { and } \\
{ }^{123} \mathrm{I} \text {-IMP }\end{array}$ & Not in daily practice & Yes & \\
\hline $\begin{array}{l}\text { Including for low } \\
\text { perfused areas }\end{array}$ & Yes & Not applicable & Not applicable & $\begin{array}{c}\text { Not below } \\
10 \mathrm{~mL} / \mathrm{min} / 100 \mathrm{~g}\end{array}$ & Yes \\
\hline Reproducibility & $5 \%$ & $10 \%$ & $10-15 \%$ & $10 \%$ & \\
\hline Spatial resolution & $4-6 \mathrm{~mm}$ & $1 \mathrm{~cm}$ & $2 \mathrm{~mm}$ & $2 \mathrm{~mm}$ & \\
\hline $\begin{array}{l}\text { Minimal time interval } \\
\text { between } 2 \text { successive } \\
\text { exams }\end{array}$ & $10 \mathrm{~min}$ & $10 \mathrm{~min}$ & $25 \mathrm{~min}$ & $0 \mathrm{~min}$ & \\
\hline $\begin{array}{l}\text { Applications in } \\
\text { neurodegenerative } \\
\text { disorders }\end{array}$ & Yes & Yes & No & Yes & Yes \\
\hline
\end{tabular}

the ASL techniques measure CBF, with the advantage of not requiring contrast injection. Many researchers are now migrating towards the use of ASL, since it poses less risk for the patients than DSC and nuclear medicine techniques.

A variety of studies in both animal models and human subjects have demonstrated that regional CBF maps can be accurately depicted by ASL [48-50], and the results obtained with ASL are consistent with those obtained from PET studies [51], since CBF and metabolic consumption are markers of cerebral dysfunction [50].

In clinical studies of $\mathrm{AD}$ and $\mathrm{MCI}$, metabolic imaging with FDG-PET $[52,53]$ and SPECT $[54,55]$ have highlighted loss of activity in temporal, parietal, and frontal association cortex, along with PCC and precuneus. Additionally, a study using a covariance ${ }^{15} \mathrm{O}$-PET pattern [56] acquired during rest showed increased flow in the insula, cuneus, pulvinar, lingual, fusiform, superior occipital, and parahippocampal gyri, whereas decreased flow was found in cingulate, inferior parietal lobe, middle and inferior frontal, and supramarginal and precentral gyri. These results are in agreement with the findings reported in ASL studies.

In $\mathrm{AD}$, medial temporal lobe is characterized by severe atrophy, so the detection of metabolism changes or subtle flow disturbances are difficult to evaluate with nuclear medicine techniques [57-59], since PET or SPECT studies do not correct for atrophy. Thus, one important advantage of combining ASL with structural MRI is the possibility of quantifying perfusion changes per $\mathrm{cc}$ of tissue in the same setting, allowing structure-function correlations within a single modality. Additionally, PET studies are prone to underestimate flow in regions with high blood flow, whereas ASL is less affected by water permeability due to the timescales of imaging and tracers decay [60]. Finally, SPECT can also suffer from saturation effects in the uptake of the tracer [61], and it has a low spatial resolution.

\section{What Can ASL Add to the Diagnosis Flow of Patients with AD in the Amyloid-PET Era?}

CSF studies and amyloid-PET imaging are considered molecular biomarkers of the disease, demonstrating the presence of the pathogenic protein "in vivo." Its detection, in one way or another, is becoming increasingly important for establishing the diagnosis of probable AD [62]. However, recent evidence shows "positive" amyloid-PET studies in cognitively healthy individuals; thus these findings should be interpreted with caution [63]. Furthermore, it involves significant costs beyond the reach of most centers. On the other hand, ASL should be considered as functional or indirect biomarker, such as FDG-PET, showing focal abnormalities in perfusion within specific brain areas due to an underlying neurodegenerative process disrupting the neurovascular coupling [24]. Thus, amyloid-PET and ASL provide different but complementary information, which ideally might be integrated together in the diagnosis process of patients with cognitive decline. However, amyloid-PET requires great economic investment, which cannot often be carried out. 


\section{Limitations of the ASL Technique in Patients with MCI/Dementia}

Discrepancies observed in different studies might be attributable to a large number of factors. First of all, there are significant differences in patients selection criteria, demographics, inclusion of other dementia syndromes, severity of the disease, neuropsychological tests used, and so forth. That is, the first study showing a hyperperfusion in the hippocampus included AD patients with unspecified severity and a mean age of $75.6 \pm 9.2$ years [9], whereas Asllani et al. study involved mild AD subjects with a mean age of $70.7 \pm 8.7$ years and Bron et al. subjects with different dementia syndromes, $\mathrm{AD}$ and frontotemporal lobe dementia, in presenile stages [15]. Secondly, methodological approaches are far from being standardized yet. Small differences between the commercial implementation of software or hardware from major MRI vendors may lead to a larger effect on the reproducibility of ASL [64]. Also, the different ASL techniques (continuous, pulsed, and pseudocontinuous), the wide range of acquisition parameters, and the different methodologies for data processing have led to an overabundance of options that has been translated in a lack of uniformity in results. Regarding acquisition parameters, most studies have used single postlabeling delay (PLD), whereas currently multiplePLD is strongly recommended for AD [65]. Whereas a large proportion apply whole brain analysis (with or without atrophy correction), some works applied regions of interest (ROI) analysis $[8,15]$.

Finally, all these studies were interpreted using visual analysis; thus the subjective component is also a potential bias. This might be overcome in the future through different techniques of CBF quantification [23]. As mentioned before, a consensus statement on recommended implementation has been recently published for helping the clinical community to adopt a standardized approach on this technique, encouraging the use of pseudocontinuous labeling, background suppression, a segmented three-dimensional readout without vascular crushing gradients, and calculation and presentation of both label/control difference images and cerebral blood flow in absolute units using a simplified model [25].

Other potential drawbacks of ASL are the relatively low SNR, a problem which can be amplified in the clinical population because of lack of cooperation, vascular disease, and artifacts. Otherwise, assumptions regarding tagging efficiency, delay time to imaging, and flow quantification are based on predominantly normal populations in the research setting, and their translation to disease states has not been rigorously tested. Additional investigations are needed to define the impact of heart rate and cardiac output variations on image quality as well as how factors such as anemia, arterial stenosis, neurofibrillary tangles, vascular amyloid deposits, and atrophy may affect well-accepted parameters such as the $\mathrm{T} 1$ of blood used to quantify CBF. Coincidentally, some of the regions with the slowest arrival are those that also show flow decreases in $\mathrm{AD}$, such as parietal and frontal association cortex. In third place, it can be challenging to obtain a good image quality in key regions for dementia research, characterized by strong magnetic susceptibility gradients (such as the inferior temporal lobes, the orbitofrontal cortex, and even the anterior medial temporal lobes), due to the proximity of these regions to air-tissue or bone-tissue interfaces [3]. Spin echo based sequences are particularly nonsensitive to these nonuniform magnetic fields $[9,66]$. Finally, because of obstacles related to licensing as well as analysis of the ASL data, it has seen little in the way of routine application in clinical populations [67]. For these reasons, large clinical cohorts are needed to define the ranges of tissue-specific perfusion, researchers should strive to employ identical parameters, and quantification methods should be implemented to avoid bias of individual visual interpretations.

\section{Conclusions}

Brain perfusion measurement is a powerful neuroimaging tool for characterizing functional brain changes that occur early in the course of AD. The detection of a hypoperfusion in the PCC and precuneus seems to be the most consistent finding through different stages of the disease (and could potentially been used as a functional biomarker of $\mathrm{AD}$ ), whereas a hyperperfusion depicted by mesial temporal lobe structures is more controversial. Current findings may provide new insights into AD pathophysiology, since these areas of hypo or hyperperfusion are promising regions for an early detection of this devastating disorder. Additionally, the ability to obtain regional CBF maps using a noninvasive and widely available modality such as ASL should greatly enhance the utility of blood flow measures as a means of gaining further knowledge into the broad range of hemodynamically related changes taking place during the cognitive decline process in $\mathrm{AD}$.

\section{Competing Interests}

The author has no conflict of interests to report.

\section{References}

[1] A.-M. Tolppanen, H. Taipale, T. Purmonen, M. Koponen, H. Soininen, and S. Hartikainen, "Hospital admissions, outpatient visits and healthcare costs of community-dwellers with Alzheimer's disease," Alzheimer's and Dementia, vol. 11, no. 8, pp. 955-963, 2015.

[2] R. C. Petersen, "Mild cognitive impairment as a diagnostic entity," Journal of Internal Medicine, vol. 256, no. 3, pp. 183-194, 2004.

[3] D. C. Alsop, W. Dai, M. Grossman, and J. A. Detre, "Arterial spin labeling blood flow MRI: its role in the early characterization of Alzheimer's disease," Journal of Alzheimer's Disease, vol. 20, no. 3, pp. 871-880, 2010.

[4] C. A. Raji, C. Lee, O. L. Lopez et al., "Initial experience in using continuous arterial spin-labeled MR imaging for early detection of Alzheimer disease," American Journal of Neuroradiology, vol. 31, no. 5, pp. 847-855, 2010.

[5] T. A. Sandson, M. O'Connor, R. A. Sperling, R. R. Edelman, and S. Warach, "Noninvasive perfusion MRI in Alzheimer's disease: 
a preliminary report," Neurology, vol. 47, no. 5, pp. 1339-1342, 1996.

[6] D. C. Alsop, J. A. Detre, and M. Grossman, "Assessment of cerebral blood flow in Alzheimer's disease by spin-labeled magnetic resonance imaging," Annals of Neurology, vol. 47, no. 1, pp. 93-100, 2000.

[7] N. A. Johnson, G.-H. Jahng, M. W. Weiner et al., "Pattern of cerebral hypoperfusion in Alzheimer disease and mild cognitive impairment measured with arterial spin-labeling MR imaging: initial experience," Radiology, vol. 234, no. 3, pp. 851-859, 2005.

[8] I. Asllani, C. Habeck, N. Scarmeas, A. Borogovac, T. R. Brown, and Y. Stern, "Multivariate and univariate analysis of continuous arterial spin labeling perfusion MRI in Alzheimer's disease," Journal of Cerebral Blood Flow and Metabolism, vol. 28, no. 4, pp. 725-736, 2008.

[9] D. C. Alsop, M. Casement, C. De Bazelaire, T. Fong, and D. Z. Press, "Hippocampal hyperperfusion in Alzheimer's disease," NeuroImage, vol. 42, no. 4, pp. 1267-1274, 2008.

[10] W. Dai, O. L. Lopez, O. T. Carmichael, J. T. Becker, L. H. Kuller, and H. M. Gach, "Mild cognitive impairment and Alzheimer disease: patterns of altered cerebral blood flow at MR imaging," Radiology, vol. 250, no. 3, pp. 856-866, 2009.

[11] A. S. Fleisher, K. M. Podraza, K. J. Bangen et al., "Cerebral perfusion and oxygenation differences in Alzheimer's disease risk," Neurobiology of Aging, vol. 30, no. 11, pp. 1737-1748, 2009.

[12] T. Yoshiura, A. Hiwatashi, K. Yamashita et al., "Simultaneous measurement of arterial transit time, arterial blood volume, and cerebral blood flow using arterial spin-labeling in patients with Alzheimer disease," American Journal of Neuroradiology, vol. 30, no. 7, pp. 1388-1393, 2009.

[13] M. A. A. Binnewijzend, J. P. A. Kuijer, M. R. Benedictus et al., "Cerebral blood flow measured with 3D pseudocontinuous arterial spin-labeling MR imaging in alzheimer disease and mild cognitive impairment: a marker for disease severity," Radiology, vol. 267, no. 1, pp. 221-230, 2013.

[14] B. Ding, H.-W. Ling, Y. Zhang et al., "Pattern of cerebral hyperperfusion in Alzheimer's disease and amnestic mild cognitive impairment using voxel-based analysis of 3D arterial spinlabeling imaging: initial experience," Clinical Interventions in Aging, vol. 9, pp. 493-500, 2014.

[15] E. E. Bron, R. M. E. Steketee, G. C. Houston et al., "Diagnostic classification of arterial spin labeling and structural MRI in presenile early stage dementia," Human Brain Mapping, vol. 35, no. 9, pp. 4916-4931, 2014.

[16] A. Xekardaki, C. Rodriguez, M.-L. Montandon et al., "Arterial spin labeling may contribute to the prediction of cognitive deterioration in healthy elderly individuals," Radiology, vol. 274, no. 2, pp. 490-499, 2015.

[17] J. A. Detre, J. S. Leigh, D. S. Williams, and A. P. Koretsky, "Perfusion imaging," Magnetic Resonance in Medicine, vol. 23, no. 1, pp. 37-45, 1992.

[18] F. Calamante, D. L. Thomas, G. S. Pell, J. Wiersma, and R. Turner, "Measuring cerebral blood flow using magnetic resonance imaging techniques," Journal of Cerebral Blood Flow and Metabolism, vol. 19, no. 7, pp. 701-735, 1999.

[19] K. Zhang, H. Herzog, J. Mauler et al., "Comparison of cerebral blood flow acquired by simultaneous $\left[{ }^{15} \mathrm{O}\right]$ water positron emission tomography and arterial spin labeling magnetic resonance imaging," Journal of Cerebral Blood Flow \& Metabolism, vol. 34, no. 8, pp. 1373-1380, 2014.
[20] S. Gevers, M. J. Van Osch, R. P. H. Bokkers et al., "Intra-and multicenter reproducibility of pulsed, continuous and pseudocontinuous arterial spin labeling methods for measuring cerebral perfusion," Journal of Cerebral Blood Flow and Metabolism, vol. 31, no. 8, pp. 1706-1715, 2011.

[21] E. T. Petersen, K. Mouridsen, and X. Golay, “The QUASAR reproducibility study, Part II: results from a multi-center Arterial Spin Labeling test-retest study," NeuroImage, vol. 49, no. 1, pp. 104-113, 2010.

[22] D. S. Williams, J. A. Detre, J. S. Leigh, and A. P. Koretsky, "Magnetic resonance imaging of perfusion using spin inversion of arterial water," Proceedings of the National Academy of Sciences of the United States of America, vol. 89, pp. 212-216, 1992.

[23] S. Verclytte, R. Lopes, P. Lenfant et al., "Cerebral hypoperfusion and hypometabolism detected by arterial spin labeling MRI and FDG-PET in Early-onset Alzheimer's disease," Journal of Neuroimaging, vol. 26, no. 2, pp. 207-212, 2016.

[24] D. Tosun, N. Schuff, W. Jagust, and M. W. Weiner, "Discriminative power of arterial spin labeling magnetic resonance imaging and 18F-fluorodeoxyglucose positron emission tomography changes for amyloid- $\beta$-positive subjects in the Alzheimer's disease continuum," Neurodegenerative Diseases, vol. 16, no. 12, pp. 87-94, 2016.

[25] D. C. Alsop, J. A. Detre, X. Golay et al., "Recommended implementation of arterial spin-labeled Perfusion mri for clinical applications: a consensus of the ISMRM Perfusion Study group and the European consortium for ASL in dementia," Magnetic Resonance in Medicine, vol. 73, no. 1, pp. 102-116, 2015.

[26] H. Lu, C. Clingman, X. Golay, and P. C. M. Van Zijl, "Determining the longitudinal relaxation time (T1) of blood at 3.0 tesla," Magnetic Resonance in Medicine, vol. 52, no. 3, pp. 679-682, 2004.

[27] M. Wintermark, M. Sesay, E. Barbier et al., "Comparative overview of brain perfusion imaging techniques," Journal of neuroradiology. Journal de neuroradiologie, vol. 32, no. 5, pp. 294-314, 2005.

[28] T. Yoshiura, A. Hiwatashi, T. Noguchi et al., "Arterial spin labelling at 3-T MR imaging for detection of individuals with Alzheimer's disease," European Radiology, vol. 19, no. 12, pp. 2819-2825, 2009.

[29] G. Xu, P. G. Antuono, J. Jones et al., "Perfusion fMRI detects deficits in regional CBF during memory-encoding tasks in MCI subjects," Neurology, vol. 69, no. 17, pp. 1650-1656, 2007.

[30] L. L. Chao, J. Pa, A. Duarte et al., "Patterns of cerebral hypoperfusion in amnestic and dysexecutive MCI," Alzheimer Disease and Associated Disorders, vol. 23, no. 3, pp. 245-252, 2009.

[31] W. Chen, X. Song, S. Beyea, R. D’Arcy, Y. Zhang, and K. Rockwood, "Advances in perfusion magnetic resonance imaging in Alzheimer's disease," Alzheimer's and Dementia, vol. 7, no. 2, pp. 185-196, 2011.

[32] B. S. Desai, J. A. Schneider, J.-L. Li, P. M. Carvey, and B. Hendey, "Evidence of angiogenic vessels in Alzheimer's disease," Journal of Neural Transmission, vol. 116, no. 5, pp. 587-597, 2009.

[33] B. P. Austin, V. A. Nair, T. B. Meier et al., "Effects of hypoperfusion in Alzheimer's disease," Journal of Alzheimer's Disease, vol. 26, supplement 3, pp. 123-133, 2011.

[34] M. Mazza, G. Marano, G. Traversi, P. Bria, and S. Mazza, "Primary cerebral blood flow deficiency and Alzheimer's disease: shadows and lights," Journal of Alzheimer's Disease, vol. 23, no. 3, pp. 375-389, 2011. 
[35] Q. Zhang, R. B. Stafford, Z. Wang, S. E. Arnold, D. A. Wolk, and J. A. Detre, "Microvascular perfusion based on arterial spin labeled perfusion MRI as a measure of vascular risk in Alzheimer's disease," Journal of Alzheimer's Disease, vol. 32, no. 3, pp. 677-687, 2012.

[36] T. Espeseth, L. T. Westlye, A. M. Fjell, K. B. Walhovd, H. Rootwelt, and I. Reinvang, "Accelerated age-related cortical thinning in healthy carriers of apolipoprotein E $\varepsilon 4$," Neurobiology of Aging, vol. 29, no. 3, pp. 329-340, 2008.

[37] M. D. Ikonomovic, E. J. Mufson, J. Wuu, E. J. Cochran, D. A. Bennett, and S. T. DeKosky, "Cholinergic plasticity in hippocampus of individuals with mild cognitive impairment: correlation with Alzheimer's neuropathology," Journal of Alzheimer's Disease, vol. 5, no. 1, pp. 39-48, 2003.

[38] S. T. Dekosky, M. D. Ikonomovic, S. D. Styren et al., "Upregulation of choline acetyltransferase activity in hippocampus and frontal cortex of elderly subjects with mild cognitive impairment," Annals of Neurology, vol. 51, no. 2, pp. 145-155, 2002.

[39] A. H. Vagnucci Jr. and W. W. Li, "Alzheimer's disease and angiogenesis," Lancet, vol. 361, no. 9357, pp. 605-608, 2003.

[40] H. Braak and E. Braak, "Frequency of stages of Alzheimerrelated lesions in different age categories," Neurobiology of Aging, vol. 18, no. 4, pp. 351-357, 1997.

[41] E. Boscolo, M. Folin, B. Nico et al., " $\beta$ amyloid angiogenic activity in vitro and in vivo," International Journal of Molecular Medicine, vol. 19, no. 4, pp. 581-587, 2007.

[42] S. L. Siedlak, P. Cras, M. Kawai, P. Richey, and G. Perry, "Basic fibroblast growth factor binding is a marker for extracellular neurofibrillary tangles in Alzheimer disease," Journal of Histochemistry and Cytochemistry, vol. 39, no. 7, pp. 899-904, 1991.

[43] C. Schultheiss, B. Blechert, F. C. Gaertner et al., "In vivo characterization of endothelial cell activation in a transgenic mouse model of Alzheimer's disease," Angiogenesis, vol. 9, no. 2, pp. 59-65, 2006.

[44] A. I. Pogue and W. J. Lukiw, "Angiogenic signaling in Alzheimer's disease," NeuroReport, vol. 15, no. 9, pp. 1507-1510, 2004.

[45] A. Naldini and F. Carraro, "Role of inflammatory mediators in angiogenesis," Current Drug Targets: Inflammation and Allergy, vol. 4, no. 1, pp. 3-8, 2005.

[46] L. Thirumangalakudi, P. G. Samany, A. Owoso, B. Wiskar, and P. Grammas, "Angiogenic proteins are expressed by brain blood vessels in Alzheimer's disease," Journal of Alzheimer's Disease, vol. 10, no. 1, pp. 111-118, 2006.

[47] P. T. Fox and M. E. Raichle, "Focal physiological uncoupling of cerebral blood flow and oxidative metabolism during somatosensory stimulation in human subjects," Proceedings of the National Academy of Sciences of the United States of America, vol. 83, no. 4, pp. 1140-1144, 1986.

[48] A. C. Silva, W. Zhang, D. S. Williams, and A. P. Koretsky, "Multi-slice MRI of rat brain perfusion during amphetamine stimulation using arterial spin labeling," Magnetic Resonance in Medicine, vol. 33, no. 2, pp. 209-214, 1995.

[49] W. Zhang, A. C. Silva, D. S. Williams, and A. P. Koretsky, "NMR measurement of perfusion using arterial spin labeling without saturation of macromolecular spins," Magnetic Resonance in Medicine, vol. 33, no. 3, pp. 370-376, 1995.

[50] J. A. Detre and D. C. Alsop, "Perfusion magnetic resonance imaging with continuous arterial spin labeling: methods and clinical applications in the central nervous system," European Journal of Radiology, vol. 30, no. 2, pp. 115-124, 1999.
[51] L. Ostergaard, D. F. Smith, P. Vestergaard-Poulsen et al., "Absolute cerebral blood flow and blood volume measured by magnetic resonance imaging bolus tracking: comparison with positron emission tomography values," Journal of Cerebral Blood Flow \& Metabolism, vol. 18, pp. 425-432, 1998.

[52] R. P. Friedland, T. F. Budinger, E. Ganz et al., "Regional cerebral metabolic alterations in dementia of the alzheimer type: positron emission tomography with $\left[{ }^{18} \mathrm{~F}\right]$ fluorodeoxyglucose," Journal of Computer Assisted Tomography, vol. 7, no. 4, pp. 590598, 1983.

[53] E. M. Reiman, R. J. Caselli, L. S. Yun et al., "Preclinical evidence of Alzheimer's disease in persons homozygous for the $\varepsilon 4$ allele for apolipoprotein E," New England Journal of Medicine, vol. 334, no. 12, pp. 752-758, 1996.

[54] F. J. Bonte, M. F. Weiner, E. H. Bigio, C. L. White III, J. M. Hoffman, and R. E. Coleman, "SPECT imaging in dementias," Journal of Nuclear Medicine, vol. 42, no. 7, pp. 1131-1133, 2001.

[55] K. A. Johnson, K. Jones, B. L. Holman et al., "Preclinical prediction of Alzheimer's disease using SPECT," Neurology, vol. 50, no. 6, pp. 1563-1571, 1998.

[56] N. Scarmeas, C. G. Habeck, E. Zarahn et al., "Covariance PET patterns in early Alzheimer's disease and subjects with cognitive impairment but no dementia: utility in group discrimination and correlations with functional performance," NeuroImage, vol. 23, no. 1, pp. 35-45, 2004.

[57] S. De Santi, M. J. De Leon, H. Rusinek et al., "Hippocampal formation glucose metabolism and volume losses in MCI and AD," Neurobiology of Aging, vol. 22, no. 4, pp. 529-539, 2001.

[58] K. Ishii, M. Sasaki, S. Yamaji, S. Sakamoto, H. Kitagaki, and E. Mori, "Paradoxical hippocampus perfusion in mild-tomoderate Alzheimer's disease," Journal of Nuclear Medicine, vol. 39, no. 2, pp. 293-298, 1998.

[59] G. Rodriguez, P. Vitali, P. Calvini et al., "Hippocampal perfusion in mild Alzheimer's disease," Psychiatry Research, vol. 100, no. 2, pp. 65-74, 2000.

[60] R. B. Buxton, L. R. Frank, E. C. Wong, B. Siewert, S. Warach, and R. R. Edelman, "A general kinetic model for quantitative perfusion imaging with arterial spin labeling," Magnetic Resonance in Medicine, vol. 40, no. 3, pp. 383-396, 1998.

[61] T. Tsuchida, Y. Yonekura, S. Nishizawa et al., "Nonlinearity correction of brain perfusion SPECT based on permeabilitysurface area product model," Journal of Nuclear Medicine, vol. 37, no. 7, pp. 1237-1241, 1996.

[62] M. S. Albert, S. T. DeKosky, D. Dickson et al., "The diagnosis of mild cognitive impairment due to Alzheimer's disease: recommendations from the National Institute on AgingAlzheimer's Association workgroups on diagnostic guidelines for Alzheimer's disease," Alzheimer's and Dementia, vol. 7, no. 3, pp. 270-279, 2011.

[63] C. M. Clark, M. J. Pontecorvo, T. G. Beach et al., "Cerebral PET with florbetapir compared with neuropathology at autopsy for detection of neuritic amyloid- $\beta$ plaques: a prospective cohort study," The Lancet Neurology, vol. 11, no. 8, pp. 669-678, 2012.

[64] H. J. M. M. Mutsaerts, M. J. P. van Osch, F. O. Zelaya et al., "Multi-vendor reliability of arterial spin labeling perfusion MRI using a near-identical sequence: implications for multi-center studies," NeuroImage, vol. 113, pp. 143-152, 2015.

[65] Y. Liu, X. Zeng, Z. Wang, N. Zhang, D. Fan, and H. Yuan, "Different post label delay cerebral blood flow measurements in patients with Alzheimer's disease using 3D arterial spin labeling," Magnetic Resonance Imaging, vol. 33, no. 9, pp. 1019$1025,2015$. 
[66] J. Wang, L. Li, A. C. Roc et al., "Reduced susceptibility effects in perfusion $\mathrm{fMRI}$ with single-shot spin-echo EPI acquisitions at 1.5 Tesla," Magnetic Resonance Imaging, vol. 22, no. 1, pp. 1-7, 2004.

[67] A. R. Deibler, J. M. Pollock, R. A. Kraft, H. Tan, J. H. Burdette, and J. A. Maldjian, "Arterial spin-labeling in routine clinical practice, part 1: technique and artifacts," American Journal of Neuroradiology, vol. 29, no. 7, pp. 1228-1234, 2008. 


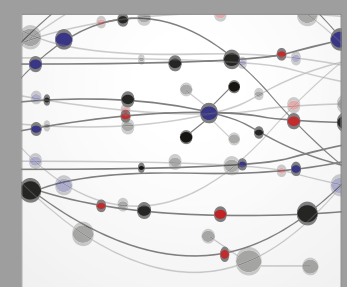

The Scientific World Journal
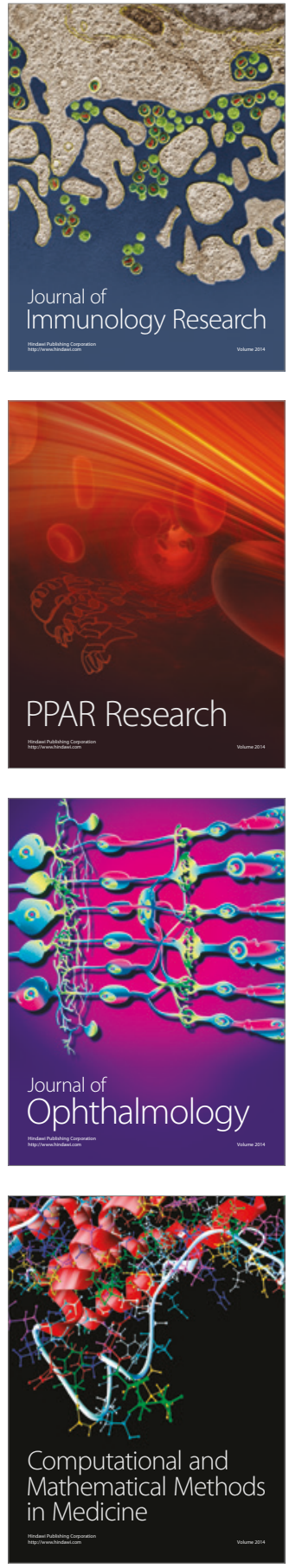

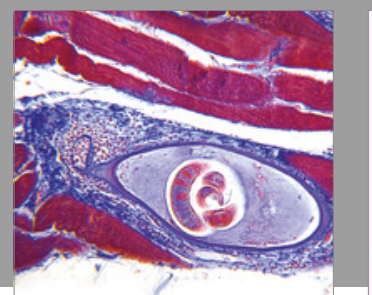

Gastroenterology Research and Practice
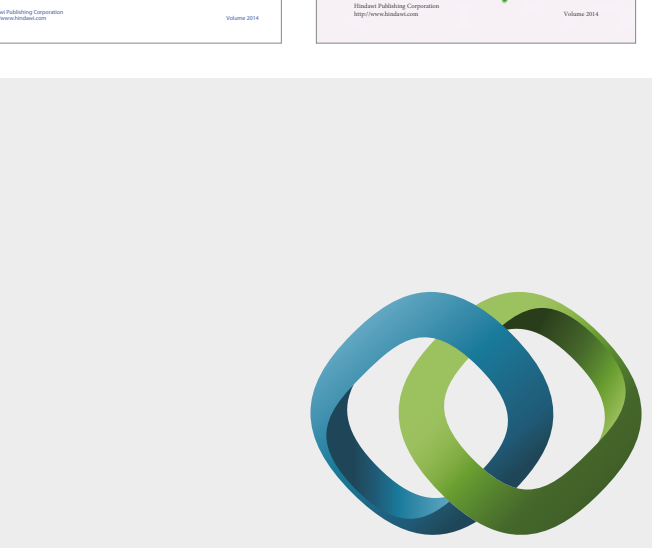

\section{Hindawi}

Submit your manuscripts at

https://www.hindawi.com
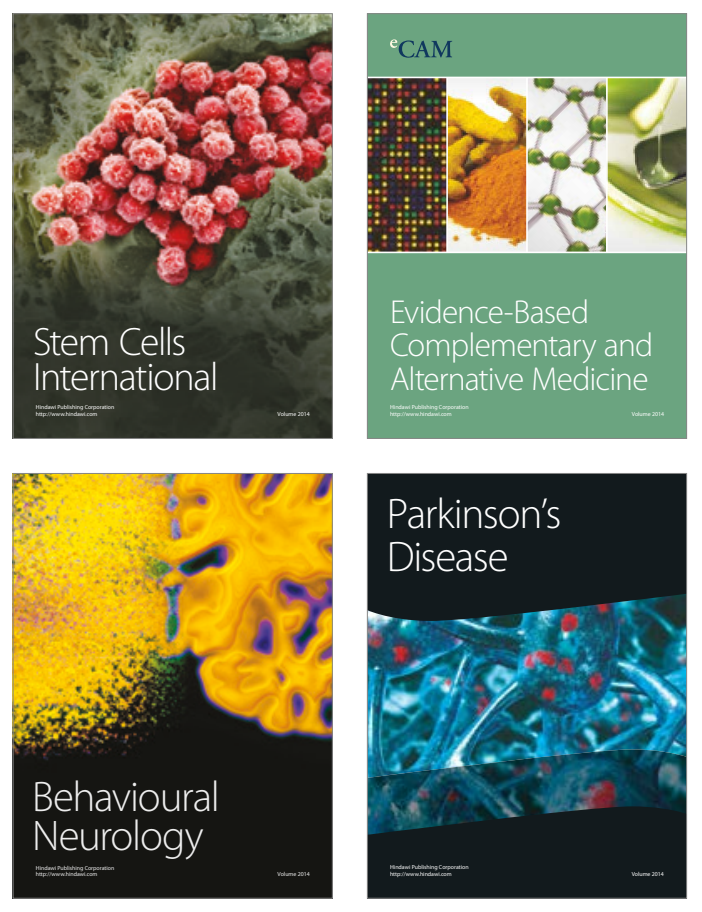
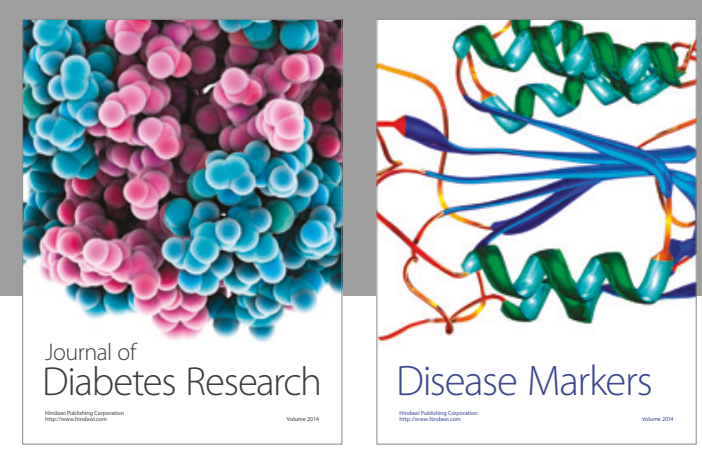

Disease Markers
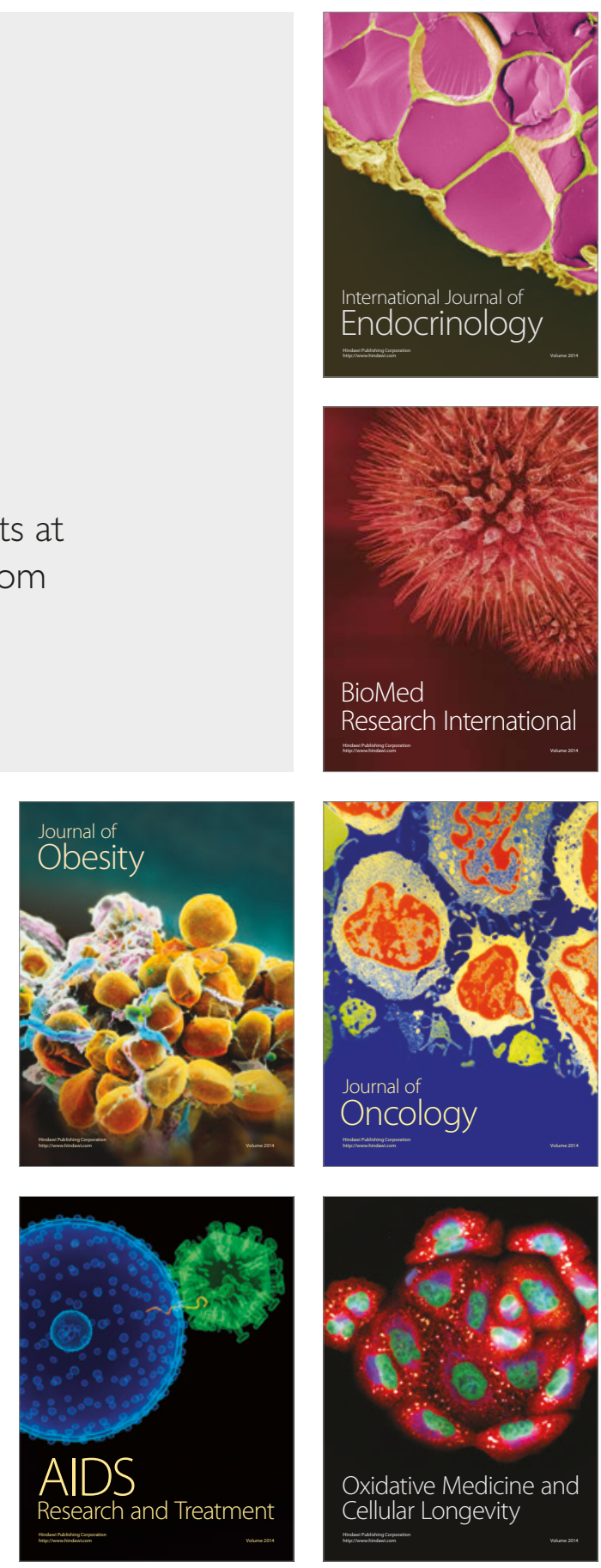